\title{
NUEVOS REGISTROS Y DISTRIBUCIÓN DE POLIQUETOS BENTÓNICOS SUBMAREALES DE FONDOS BLANDOS EN LA REGIÓN DE AISÉN, CHILE: FAMILIAS PARAONIDAE Y SPIONIDAE (ANNELIDA: POLYCHAETA).
}

\author{
NEW RECORDS AND DISTRIBUTION OF BENTHIC SUBTIDAL \\ POLYCHAETES ON SOFT BOTTOM IN AISÉN, CHILE: FAMILIES \\ PARAONIDAE AND SPIONIDAE (ANNELIDA: POLYCHAETA).
}

Paula Ortiz ${ }^{1} \&$ Eduardo Quiroga ${ }^{2}$

\begin{abstract}
Results from a taxonomic analysis of subtidal benthic polychaetes belonging to the Paraonidae and Spionidae families are present. The samples were taken during benthic research cruises, conducted by the Center for Advance Research in Patagonian Ecosystems (CIEP), at the Aisén region (Northern Patagonia). Benthic samples were collected from the Piti-Palena fjord, Aisén fjord, Estero Elefantes and Baker fjord, between 6 and $126 \mathrm{~m}$ water depth. We identified five species of Paraonidae family (Aricidea antartica Hartmann-Schröder \& Rosenfeldt 1988, Aricidea strelzovi Hartmann-Schröder \& Rosenfeldt 1990, Aricidea catherinae Laubier 1967, Aricidea albatrossea Pettibone 1957 and Levinsenia antartica (Strelzov 1973)) and nine species of Spionidae family (Spiophanes soederstroemi Hartman 1953, Spiophanes bombyx (Claparede 1870), Scolelepis chilensis Hartmann-Schröder 1962, Rhynchospio glutaea (Ehlers 1897), Prionospio patagonica Augener 1923, Prionospio elhersi Fauvel 1928, Prionospio orensanzi Blake 1983, Laonice weddellia Hartman 1978 and Aonides sp). New records and range extensions of paranoids and spionids polychaetes species in the Aisen region and Chile are reported.
\end{abstract}

Key words: Polychaeta, Paraonidae, Spionidae, Aisén Region, Chile.

1 Centro de Investigación en Ecosistemas de la Patagonia (CIEP), Bilbao 449, Coyhaique, Chile. paulaortiz@ciep.cl (P. Ortiz).

2 Pontificia Universidad Católica de Valparaíso (PUCV), Escuela de Ciencias del Mar, Casilla 1020, Av. Altamirano 1480, Valparaíso, Chile. 


\section{RESUMEN}

Se presentan los resultados del análisis taxonómico de poliquetos bentónicos de las familias Paraonidae y Spionidae en fondos blandos submareales provenientes de diversas campañas de investigación conducidas por el Centro de Investigación en Ecosistemas de la Patagonia (CIEP) en la región de Aisén, Chile. Las muestras fueron recolectadas en el fiordo Piti-Palena, fiordo Aisén, estero Elefantes y fiordo Baker, entre los 6 y 126 m de profundidad. Durante estos estudios se han identificado cinco especies de poliquetos de la familia Paraonidae (Aricidea antartica Hartmann-Schröder \& Rosenfeldt, 1988, Aricidea strelzovi Hartmann-Schröder \& Rosenfeldt, 1990, Aricidea catherinae Laubier, 1967, Aricidea albatrossea Pettibone, 1957 y Levinsenia antartica (Strelzov, 1973)) y nueve especies de la familia Spionidae (Spiophanes soederstroemi Hartman, 1953, Spiophanes bombyx (Claparède, 1870), Scolelepis chilensis Hartmann-Schröder, 1962, Rhynchospio glutaea (Ehlers, 1897), Prionospio patagonica Augener, 1923, Prionospio elhersi Fauvel, 1928, Prionospio orensanzi Blake, 1983, Laonice weddellia Hartman, 1978 y Aonides sp). Se reportan nuevos registros y extensión de los rangos de distribución de estas especies de paraonidos y espionidos en la región de Aisén y Chile.

Palabras clave: Poliquetos, Paraonidae, Spionidae, Región de Aisén, Chile.

\section{INTRODUCCION}

La región Sur Austral de Chile está conformada por numerosas islas, fiordos y canales, cuya extensión geográfica es de aproximadamente $84.000 \mathrm{Km}$ de costas interiores, lo cual resulta de gran interés científico para el estudio de la diversidad marina (Häussermann \& Försterra 2009). Sin embargo existe un número reducido de estudios que consideren aspectos relacionados con la diversidad, siendo esta información de gran importancia para los programas de vigilancia ambiental en el marco de la legislación vigente para la actividad de salmonicultura debido a la fuerte presión por el desarrollo de la actividad de salmonicultura en la región (Buschmann et al. 2006). En este contexto, las investigaciones sobre las familias Paraonidae y Spionidae de la región Sur Austral de Chile es restringida a solo algunas zonas geográficas de la región de Aisén (Cañete et al. 1999) y en la región de Magallanes (Montiel et al. 2002, 2004, 2005). La fauna de poliquetos bentónicos en estas regiones constituye un componente dominante de los fondos blandos submareales, cuyas especies presentan una gran variedad de estrategias tróficas, incluyendo carnívoros, herbívoros y depositívoros.

En el marco de los estudios realizados por el Centro de Investigación en Ecosistemas de la Patagonia (CIEP) en la región de Aisén, se han realizado diversas campañas científicas, cuyos resultados nos han permitido incrementar la información sobre la diversidad de los poliquetos bentónicos submareales en áreas de difícil acceso tales como el fiordo Piti-Palena, estero Elefantes, fiordo Aisén y el fiordo Baker. Estos estudios han generado nuevo conocimiento sobre los patrones de distribución de la fauna bentónica en la región. Estudios sobre aspectos taxonómicos y ecológicos han sido realizados para el fiordo Aisén y estero Elefantes (Cañete et al. 1999, Rozbaczylo et al. 2005, 2006a, 2006b, 2006c), mientras que escasos estudios han sido realizados para el fiordo Baker. Sin embargo, no se han realizado estudios sobre el componente bentónico en el fiordo PitiPalena. El presente trabajo tiene como objetivo entregar información sobre nuevos registros de especies y la distribución de las especies de poliquetos pertenecientes a las familias Paraonidae y Spionidae en la región de Aisén.

\section{MATERIALES \& MÉTODOS}

Los poliquetos fueron recolectados como parte de material faunístico provenientes de diversas campañas de investigación en la región de Aisén realizadas por el Centro de Investigación en Ecosistemas de la Patagonia (CIEP) entre el año 2006 y 2009. Las muestras fueron obtenidas en cuatro localidades:

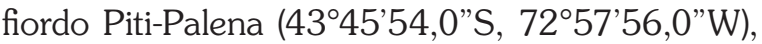
fiordo Aisén ( $45^{\circ} 14^{\prime} 34,3^{\prime \prime}$ S, $\left.73^{\circ} 13^{\prime} 36,9^{\prime \prime} \mathrm{W}\right)$, estero Elefantes $\left(45^{\circ} 23^{\prime} 51,8^{\prime \prime} S, 73^{\circ} 39^{\prime} 21,4^{\prime \prime W}\right)$ y fiordo 


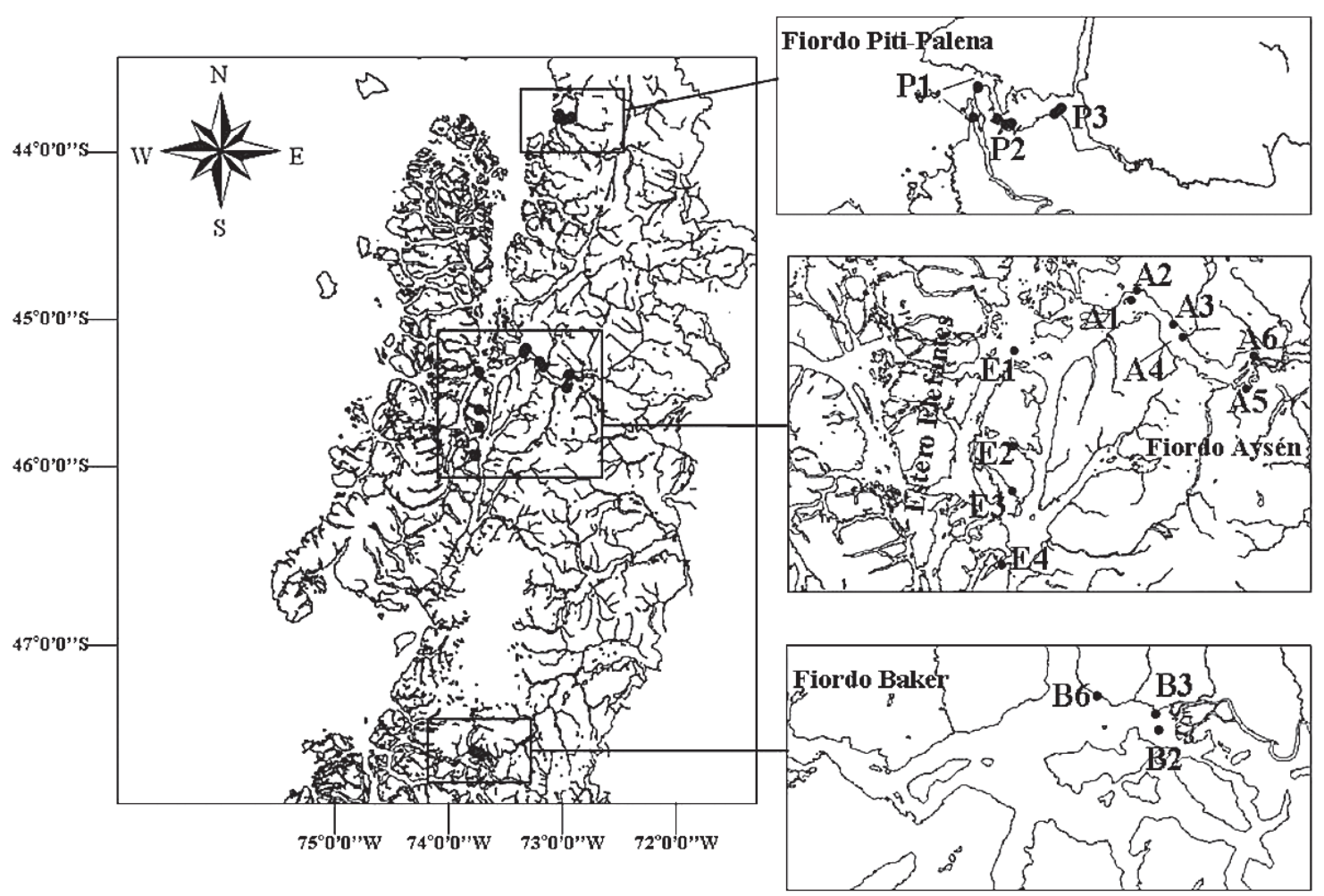

Fig. 1. Mapa del área de estudio indicando la posición de las estaciones de muestreo.

Baker (4747'10,9'S, 73³5'20,3'W) en profundidades entre 6 y $126 \mathrm{~m}$ (Fig. 1 y Tabla 1 ).

Los ejemplares fueron obtenidos de muestras de sedimentos, las cuales se colectaron a través de una draga van Veen de $0,1 \mathrm{~m}^{2}$ de área de mordida, un sacatestigo de $50 \mathrm{~mm}$ de diámetro interno. Además se colectaron ejemplares de muestras de sedimento obtenidas por medio de buceo autónomo (SCUBA) con un sacatestigo de $50 \mathrm{~mm}$ de diámetro interno. Se recolectaron 4 a 5 réplicas en cada sector de muestreo, el número de réplicas dependió de las condiciones climáticas y de la profundidad (Tabla 1). El material biológico previamente fijado con formalina en agua de mar al 4\%, se hizo pasar por un cedazo de $500 \mu \mathrm{m}$ y luego se preservó en alcohol al 70\%. El material biológico fue identificado al nivel taxonómico más bajo posible en base a la literatura disponible, utilizando una lupa binocular estereoscópica y un microscopio óptico. Para la determinación taxonómica de las familias Paraonidae y Spionidae se utilizaron los trabajos de Hartmann-Schröder (1962, 1965), Hartman (1964), Fauchald (1977),
Blake (1983), Day (1967), Hobson \& Banse (1981), Rozbaczylo (1985) y Montiel et al. (2002).

\section{RESULTADOS}

Spionidae Grube, 1850

Laonice Malgrem, 1867

Laonice weddellia Hartman, 1978

(pp.161-163, figs. 16a-16c).

Localidades citadas para Chile:

Frente a la costa, entre Los Vilos y Tongoy, Eltanin Sta.72, $31^{\circ} 06.30^{\prime}$ ' , $71^{\circ} 48.50$ 'W, 805-970 m; estrecho de Magallanes, paso Froward, Eltanin Sta.962, 256-320 m; islas Piloto Pardo, al NE de isla Clarence, Eltanin Sta.1089, 641 m; estrecho Bransfield, NE de isla D'Urville, Eltanin Sta.418, 311-426 m; estrecho Bransfield, frente a la entrada a bahía Almirantazgo, isla Rey Jorge, Eltanin Sta.419, 509-549 m; estrecho Bransfield, islas Shetland del Sur, cerca de isla Decep- 
TABLA 1, Lista de localidades, fecha de muestreo, coordenadas geográficas y rango de profundidad de las estaciones de muestreo en la región de Aisén,

\begin{tabular}{|c|c|c|c|c|c|c|}
\hline Localidad & Sector & Estación & Fecha & Lat, S & Long, W & Profundidad $(\mathrm{m})$ \\
\hline \multirow[t]{3}{*}{ Fiordo Piti-Palena } & Desembocadura Río Palena & P1 & $07,02,2006$ & $43^{\circ} 16^{\prime} 03,7^{\prime \prime}$ & $73^{\circ} 14^{\prime} 37,9^{\prime \prime}$ & $6-8$ \\
\hline & Canal Garrao & P2 & 06.02 .2006 & $43^{\circ} 47^{\prime} 59,0^{\prime \prime}$ & $72^{\circ} 05^{\prime} 36,0^{\prime \prime}$ & $12-16$ \\
\hline & Río Rodríguez & P3 & 06.02 .2006 & $43^{\circ} 47^{\prime} 05,0^{\prime \prime}$ & $72^{\circ} 51^{\prime} 11,0^{\prime \prime}$ & $6-10$ \\
\hline \multirow[t]{6}{*}{ Fiordo Aysen } & Cinco Hermanas & A1 & 14.10 .2009 & $45^{\circ} 16^{\prime} 03,7^{\prime \prime}$ & $73^{\circ} 14^{\prime} 37,9^{\prime \prime}$ & $46-50$ \\
\hline & Caleta Pérez & A2 & 14.10.2009 & $45^{\circ} 14^{\prime} 34,3^{\prime \prime}$ & $73^{\circ} 13^{\prime} 36,9^{\prime \prime}$ & $33-36$ \\
\hline & Punta Tortuga & A3 & 15.10 .2009 & $45^{\circ} 19^{\prime} 28,7^{\prime \prime}$ & $73^{\circ} 05^{\prime} 36,1^{\prime \prime}$ & $33-35$ \\
\hline & Desembocadura Río Cuervo & A4 & 15.10 .2009 & $45^{\circ} 21^{\prime} 14,4^{\prime \prime}$ & $73^{\circ} 03^{\prime} 35,9^{\prime \prime}$ & $45-50$ \\
\hline & Caleta Bluff & A5 & 16.10 .2009 & $45^{\circ} 28^{\prime} 41,6^{\prime \prime}$ & $72^{\circ} 49^{\prime} 54,3^{\prime \prime}$ & $23-26$ \\
\hline & Bahía Acantilada & A6 & 16.10 .2009 & $45^{\circ} 23^{\prime} 45,0^{\prime \prime}$ & $72^{\circ} 48^{\prime} 25,7^{\prime \prime}$ & $43-47$ \\
\hline \multirow[t]{4}{*}{ Estero Elefantes } & Paulina & E1 & 10.12 .2007 & $45^{\circ} 23^{\prime} 51,8^{\prime \prime}$ & $73^{\circ} 39^{\prime} 21,4^{\prime \prime}$ & $11-44$ \\
\hline & Nevenka & $\mathrm{E} 2$ & 10.12 .2007 & $45^{\circ} 44^{\prime} 29,6^{\prime \prime}$ & $73^{\circ} 39^{\prime} 07,5^{\prime \prime}$ & $55-126$ \\
\hline & Pescadores & E3 & 10.12.2007 & $45^{\circ} 55^{\prime} 20,7^{\prime \prime}$ & $73^{\circ} 41^{\prime} 08,1^{\prime \prime}$ & $27-67$ \\
\hline & San Ramón & E4 & 10.12.2007 & $45^{\circ} 37^{\prime} 50,3^{\prime \prime}$ & $73^{\circ} 39^{\prime} 11,6^{\prime \prime}$ & $40-43$ \\
\hline \multirow[t]{11}{*}{ Fiordo Baker } & Desembocadura Río Baker & B1 & 29.06.2008 & $47^{\circ} 47^{\prime} 53,0^{\prime \prime}$ & $73^{\circ} 35^{\prime} 05,6^{\prime \prime}$ & $40-54$ \\
\hline & $\begin{array}{l}\text { Bajo Pisagua, } \\
\text { Desembocadura Río Baker }\end{array}$ & B2 & 29.06.2008 & $47^{\circ} 47^{\prime} 10,9^{\prime \prime}$ & $73^{\circ} 35^{\prime} 20,3^{\prime \prime}$ & $29-48$ \\
\hline & $\begin{array}{l}\text { Bajo Pisagua, } \\
\text { Desembocadura Río Baker }\end{array}$ & B2 & 08.09 .2008 & $47^{\circ} 47^{\prime} 10,9^{\prime \prime}$ & $73^{\circ} 35^{\prime} 20,3^{\prime \prime}$ & $29-48$ \\
\hline & Desembocadura Río Baker & B1 & 28.11.2009 & $47^{\circ} 47^{\prime} 53,0^{\prime \prime}$ & $73^{\circ} 35^{\prime} 05,6^{\prime \prime}$ & $40-54$ \\
\hline & $\begin{array}{l}\text { Bajo Pisagua, } \\
\text { Desembocadura Río Baker }\end{array}$ & B2 & 28.11.2009 & $47^{\circ} 47^{\prime} 10,9^{\prime \prime}$ & $73^{\circ} 35^{\prime} 20,3^{\prime \prime}$ & $29-48$ \\
\hline & Desembocadura Río Baker & B1 & 24.02.2009 & $47^{\circ} 47^{\prime} 53,0^{\prime \prime}$ & $73^{\circ} 35^{\prime} 05,6^{\prime \prime}$ & $40-54$ \\
\hline & $\begin{array}{l}\text { Bajo Pisagua, } \\
\text { Desembocadura Río Baker }\end{array}$ & B2 & 24.02.2009 & $47^{\circ} 47^{\prime} 10,9^{\prime \prime}$ & $73^{\circ} 35^{\prime} 20,3^{\prime \prime}$ & $29-48$ \\
\hline & Punta Raúl & B3 & 28.06.2008 & $47^{\circ} 46^{\prime} 27,1^{\prime \prime}$ & $73^{\circ} 39^{\prime} 15,3^{\prime \prime}$ & $34-66$ \\
\hline & Punta Raúl & B3 & 08.09 .2008 & $47^{\circ} 46^{\prime} 27,1^{\prime \prime}$ & $73^{\circ} 39^{\prime} 15,3^{\prime \prime}$ & $34-66$ \\
\hline & Punta Raúl & B3 & 26.11.2008 & $47^{\circ} 46^{\prime} 27,1^{\prime \prime}$ & $73^{\circ} 39^{\prime} 15,3^{\prime \prime}$ & $34-66$ \\
\hline & Punta Raúl & B3 & 23.02.2009 & $47^{\circ} 46^{\prime} 27,1^{\prime \prime}$ & $73^{\circ} 39^{\prime} 15,3^{\prime \prime}$ & $34-66$ \\
\hline
\end{tabular}

ción, West-wind Sta.9, 62 $24^{\circ} \mathrm{S}, 59^{\circ} 44^{\prime} \mathrm{W}, 170 \mathrm{~m}$; islas Shetland del Sur, Hero Sta.726; 62 $19,3^{\prime}$ 'S, 59¹1,8'W, 62-84 m; Hero Sta.1060, y Sta.1063, 62¹9,0’S, $59^{\circ} 11,4^{\prime} \mathrm{W}, 44 \mathrm{~m}$; estrecho Bransfield, al WSW de isla Elefante, Eltanin Sta. 410, 220-246 m; estrecho Bransfield, al E de isla Rey Jorge, Eltanin Sta.412, y Sta.413, 1180 m, y 1113-1153; estrecho Bransfield, al NE de isla D'Urville, Eltanin Sta.416, 494-507 m; estrecho Bransfield, al N de isla Decepción, Eltanin Sta.437, 267-311 m; península Antártica, al W de isla Víctor Hugo, Eltanin Sta. 272, 412 m; península Antártica, frente a isla Anvers, Hero Sta.767, 64²5'S, $64^{\circ} 05^{\prime} \mathrm{W}$, buceo; archipiélago de Palmer, isla Anvers, bahía Arthur, Hero Sta.843, 64²47,5'S, 64º7,2'W, 107 m; península Antártica, estrecho Bismarck, Hero Sta.970, 6449,04'S, 63³2,8’W, 102 m; península Antártica, isla Anvers fuera de bahía Arthur, Hero Sta.1066, 64²47,4'S, 64º6,8'W; península Antártica, isla Anvers, S de bahía Arthur, Hero Sta.1075, 6447,4'S, 6407,2'W, 91-110 m; península Antártica, estrecho Bismark, Hero Sta.1937, 6452,20' a 6452,36'S, 6332,40' a 6333,22'W, 96-133 m; península Antártica, isla Anvers, bahía Arthur, International Weddell Sea Oceanographic Expedition Sta. 68 Palmer II, 40 m; Mar de Weddell, Weddell Sea Oceanographic Expedition Sta.68-5, 7600'S, 5500'W, 400 m, International Weddell Sea Oceanographic Expedition Sta.68-9, 73³1'S, 6003’W, 526 m; Fondeadero en base Palmer ("Hero Inlet"), isla Anvers, archipiélago de Palmer, 40 m (Hartman 1978, Blake 1983).

Presente estudio: fiordo Aisén, sector Caleta Bluff (4528'41,6”S, 7249'54,3'W, 23-26 m). fiordo Baker, Bajo Pisagua (4747'10,9'S, 73³5’20,3”W, $29-48 \mathrm{~m})$. 
Spiophanes Grube, 1860

Spiophanes soederstroemi Hartman, 1953 (p. 41, fig. 14 a-c).

Localidades citadas para Chile:

Coquimbo, $82 \mathrm{~m}$; punta Topocalma, $90 \mathrm{~m}$; punta Lavapié, $58 \mathrm{~m}$; NE de isla Mocha, frente a punta Nena, 26-160 m; N de punta Ronca, $71 \mathrm{~m}$ punta Galera, 84-260 m; golfo Corcovado, al E de isla Puduhuapi, $190 \mathrm{~m}$ (Marchile I, Sta.1, Sta.39, Sta.68, Sta.75, Sta.77, Sta.91, Sta.93, Sta.94, Sta.95, Sta.96, Sta.XI, Hartmann-Schröder 1965). archipiélago de Juan Fernández, Anton Bruun

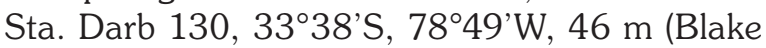

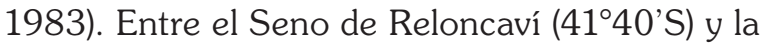

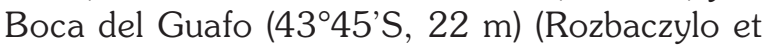
al. 2006c).

Presente estudio: fiordo Piti-Palena, sector desembocadura río Palena $\left(45^{\circ} 16^{\prime} \mathrm{S}, 73^{\circ} 14^{\prime} \mathrm{W}\right.$,

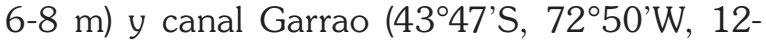
$16 \mathrm{~m})$. fiordo Aysén, sector bahía Acantilada $\left(45^{\circ} 23^{\prime} 45,0^{\prime \prime} \mathrm{S}, 72^{\circ} 48^{\prime} 25,7^{\prime \prime} \mathrm{W}, 43-47 \mathrm{~m}\right)$ y punta Tortuga $\left(45^{\circ} 19^{\prime} 28,7^{\prime \prime} \mathrm{S}, 73^{\circ} 05^{\prime} 36,1^{\prime \prime} \mathrm{W}, 33-\right.$ $35 \mathrm{~m})$. fiordo Baker, desembocadura río Baker $\left(47^{\circ} 47^{\prime} 53,0^{\prime \prime} \mathrm{S}, 73^{\circ} 35^{\prime} 05,6^{\prime \prime} \mathrm{W}, 40-54 \mathrm{~m}\right)$ y Bajo Pisagua (4747'10,9'S, $\left.73^{\circ} 35^{\prime} 20,3^{\prime \prime} \mathrm{W}, 29-48 \mathrm{~m}\right)$. estero Elefantes, sector San Ramón (4537'50,3'S, 73³9'15,3”W, 40-43 m) y Pescadores (4555'20,7'S, $\left.73^{\circ} 41^{\prime} 08,1^{\prime \prime} \mathrm{W}, 27-67 \mathrm{~m}\right)$.

Spiophanes bombyx (Claparede, 1870)

(pp. 485-487, pl. 12, fig.2)

Localidades citadas para Chile:

Bahía Coliumo (Carrasco 1974). Frente a caleta Leandro, 36³8'36"S, 7305'24"W (Carrasco 1976). Archipiélago de Palmer, isla Wiencke, puerto Lockroy, 70 m (Augener 1932).

Presente estudio: estero Elefantes, sector

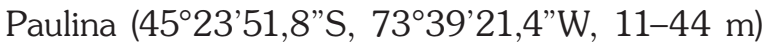
y Pescadores (455' $20,7^{\prime \prime} S, 73^{\circ} 41^{\prime} 08,1^{\prime \prime} \mathrm{W}, 27-67$ m). Fiordo Aisén, sector Caleta Bluff ( $45^{\circ} 28^{\prime} 41,6^{\prime \prime}$, $72^{\circ} 49 ' 54,3$ 'W, 23-26 m).
Prionospio Malgrem, 1867

Prionospio (Minuspio) patagonica

Augener, 1923 (pp.3-5).

Localidades citadas para Chile:

Originalmente fue descrito en el estero Kelly, Aisén (Augener 1923). Desembocadura de la quebrada de Córdova, $\mathrm{N}$ de El Tabo; cabo Quedal, 136-176 m; golfo de Ancud, SE de isla Tabón 264 $\mathrm{m}$; entre el golfo de Ancud y el golfo de Corcovado, ESE de isla Tac, $240 \mathrm{~m}$ (MarChile I, Sta.107, Sta.108, Sta.X3, Sta.X2, Hartmann-Schröder 1965). $30 \mathrm{Km}$ al S de Constitución; estero Lenga, bahía San Vicente; Niebla, Valdivia (Hartmann-Schröder 1962). Canal Beagle, entre puerto Navarino y puerto Williams, $140 \mathrm{~m}$; ribera norte del canal Beagle, entre Ushuaia y Lapataia, 35 m (Swdish Antarctic Expedition. Sta.62, Sta.64, Hartman 1953). Lago Budi (3849'30”'S, 73²3'30”W, 7 m) (Bertrán et al. 2010). Lagos Cucao (42 39'S, $\left.74^{\circ} 00^{\prime} \mathrm{W}\right)$ y Huillinco $\left(42^{\circ} 41^{\prime} \mathrm{S}, 73^{\circ} 54^{\prime} \mathrm{W}\right)$, Isla de Chiloé (Bertrán et al. 2006).

Presente estudio: Intermareal, en el delta del río Baker (4748’11,2”S, 73³3'33,4”W).

Prionospio ehlersi Fauvel, 1928 (pp.10-12, fig.1).

Localidades citadas para Chile:

Valparaíso, frente a punta Angeles, Eltanin Sta.750, 624 m; Paso Drake, al W de isla Smith, Eltanin Sta.265, 3691-3693 m, Paso Drake, Eltanin Sta.303, 62 $2^{\circ} 01^{\prime}$ a $62^{\circ} 06^{\prime} S, 70^{\circ} 59^{\prime}$ a $70^{\circ} 51^{\prime} \mathrm{W}, 4077$ $\mathrm{m}$ (Blake 1983).

Presente estudio: estero Elefantes, sector San Ramón (45³7'50,3”S, 73³9'15,3”'W, 40-43 m) y Pescadores $\left(45^{\circ} 55^{\prime} 20,7^{\prime \prime} \mathrm{S}, 73^{\circ} 41^{\prime} 08,1^{\prime \prime} \mathrm{W}\right.$, 27-67 m). Fiordo Baker, desembocadura río Baker (4747’53,0”S, 7335'05,6”'W, 40-54 m).

Prionospio orensanzi Blake, 1983

(pp.225, figs.10-11).

Localidades citadas para Chile:

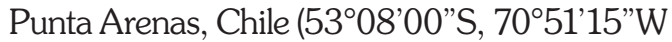

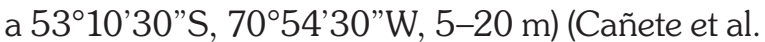
2004). Bahía de Corral (3950'52”'S, 73²7’38”W) 
y estuario Río Valdivia (3950'22'S, 73¹6’06”W) (Díaz 2004³).

Presente estudio: estero Elefantes, sector Paulina (4523'51.8'S, 73³9'21.4"W, 11-44 m)

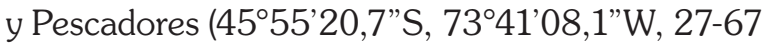
m). Fiordo Aisén, sector caleta Bluff (45 28'41,6"S, 72 $49^{\prime} 54,3^{\prime \prime}$, 23-26 m) y punta Tortuga (4519'28,7's, 7305'36,1's, 33-35 m).

Rhynchospio Hartman, 1936

Rhynchospio glutaea (Ehlers, 1897)

Localidades citadas para Chile:

Bahía Concepción (Carrasco 1974). Bahía de Concepción, en la desembocadura del río Andalién (Hartmann-Schröder 1962). Punta Lavapié, 58 m; Ahínco-Pargua, ribera $\mathrm{N}$ del canal Chacao; bahía de Ancud, puerto Inglés, $12 \mathrm{~m}$ (Mar-Chile I, Sta. 68, Hartmann-Schröder 1965). Estrecho de Magallanes, Punta Arenas, 2 brazas (Ehlers 1987, 1901). Mar de Weddell, al SW de isla Cerro Nevado, 125 m (Swedish Antarctic Expedition Sta. 6, Hartman 1953). Bahía Iquique (20¹1'35’'S, 7008'25”W), 6-22 m (Moreno et al. 2002).

Presente estudio: Estero Elefantes, sector

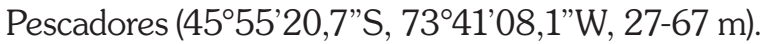

Scolelepis Blainville, 1828

Scolelepis chilensis (Hartmann-Schröder, 1962) (pp. 142-144, figs. 179-182).

Localidades citadas para Chile:

Huasco (Hartmann-Schröder, 1962). Montemar, Playa Cochoa, en arena; Mehuín, Valdivia, intermareal (Blake 1983).

Presente estudio: estero Elefantes, sector Paulina (45²3'51,8”S, 73³9'21,4”W,11-44 m).

3 Díaz, M. 2004. Aspectos morfológicos, reproductivos y desarrollo larval de Prionospio patagonica (Augener, 1923) y Prionospio orensanzi (Blake, 1983), (Polychaeta:Spionidea), presentes en el Sur de Chile. Tesis de grado para optar al título de Biólogo Marino, Facultad de Ciencias, Universidad Austral de Chile.
Aonides Claparede 1864

Aonides sp.

Localidades citadas para Chile:

No hay registros previos en aguas Chilenas del género. Se ha registrado Aonides paucibranchiata Southern 1914 en el NE atlántico, Mar del Norte, Mar Negro y Argentina (Blake 1983).

Presente estudio: fiordo Baker, desembocadura río Baker (47²7'53,0”S, 73³5’05,6”W, 40-54 m), sector Bajo Pisagua (4747'10,9'S, 73³5'20,3'W, 29-48 m) y punta Raúl (4746'27,1'S, 73³9'15,3”W, 34-66 m). Fiordo Aisén, sector Bahía Acantilada (4523'45,0'S, 7248'25,7'W, 43-47 m), caleta Bluff (4528'41,6”'S, 7249'54,3'W, 23-26 m), desembocadura río Cuervo (45²1'14,4”S, 7303'35,9”W, 45-50 m) y punta Tortuga $\left(45^{\circ} 19^{\prime} 28,7^{\prime \prime} S, 73^{\circ} 05^{\prime} 36,1^{\prime \prime} \mathrm{W}\right.$, 33-35 m).

\section{Paraonidae Cerruti 1909}

Aricidea Webster 1879

Aricidea antartica Hartmann-

Schröder \& Rosenfeldt 1988

Localidades citadas para Chile:

Región de Magallanes, Plataforma continental Tierra del Fuego 5528,8'S, 6604,4'W, 1162 m (Montiel et al. 2002).

Presente estudio: estero Elefantes, sector San Ramón (4537'50,3'S, 73³9'11,6”W, 40-43 m),

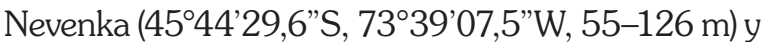
Pescadores (4555'20,7'S, 7341'08,1"W, 27-67 m). fiordo Aysén, sector bahía Acantilada (45²3’45,0"S, 7248'25,7”W, 43-47 m), caleta Bluff (4528'41,6"S, 7249'54,3"W, 23-26 m), desembocadura río Cuer-

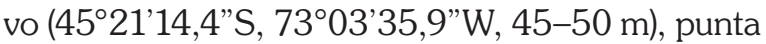
Tortuga (4519'28,7'S, $\left.73^{\circ} 05^{\prime} 36,1^{\prime \prime} \mathrm{W}, 33-35 \mathrm{~m}\right)$, caleta Pérez (45¹4'34,3"S, 73¹3'36,9”W, 33-36 m) y Cinco Hermanas (4516’03,7'S, 73¹4'37,9"W, 46-50 m). Fiordo Baker, desembocadura río Baker (4747'53,0”S, 7335'05,6”W, 40-54 m), sector Bajo Pisagua (47²7'10,9"S, 73³5'20,3'W, 29-48 m) y punta Raúl (4746’27,1”S, 73³9’15,3”W, 34-66 m). 
Aricidea strelzovi Hartmann-

Schröder \& Rosenfeldt, 1990

Localidades citadas para Chile:

Región de Magallanes, canal Picton, Campo de Hielo Sur $49^{\circ} 28,9^{\prime}$ S, $75^{\circ} 25,2^{\prime} \mathrm{W}, 98 \mathrm{~m}$ a la Plataforma continental Tierra del Fuego $55^{\circ} 28,8^{\prime} \mathrm{S}$, $66^{\circ} 04,4^{\prime} \mathrm{W}, 382 \mathrm{~m}$ (Montiel et al. 2002).

Presente estudio: fiordo Piti-Palena, sector canal Garrao (4347'59.0”S, 7205'36,0”W, 12-16 m) y Río Rodríguez (4347’05,0"S, 72 51'11,0"W, 6-10 m). Estero Elefantes, sector Paulina (4523'51,8'S, 73³9'21,4”W, 11-44 m), San Ramón (45³7'50,3”S, 73³9'11,6”W, 40-43 m), Nevenka (4544'29,6”S, 73³9'07,5”W, 55-126 m) y Pescadores $\left(45^{\circ} 55^{\prime} 20,7^{\prime} \mathrm{S}, 7^{\circ} 41^{\prime} 08,1^{\prime \prime} \mathrm{W}\right.$, 27-67 m). Fiordo Aisén, sector Cinco Hermanas (4516'03,7's, 7314'37,9”W, 46 - 50 m).

Aricidea catherinae Laubier, 1967

Localidades citadas para Chile:

Región de Magallanes, Estrecho de Magallanes, Punta Arenas 5310,2'S, 7052,3'W, $26 \mathrm{~m}$ a

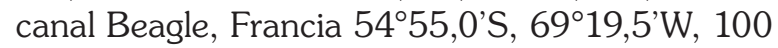
m (Montiel et al. 2002).

Presente estudio: estero Elefantes, sector Paulina (4523'51,8'S, 73³9'21,4”W, 11-44 m) y

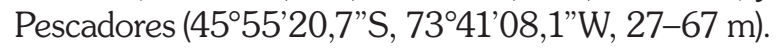

Aricidea albatrossae Pettibone, 1957

Localidades citadas para Chile:

Región de Magallanes, Plataforma Conti-

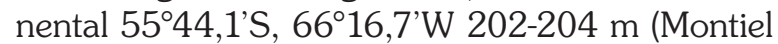
et al. 2002).

Presente estudio: estero Elefantes, sector San Ramón (45³7'50,3”S, 73³9'11,6”W, 40-43 m) y

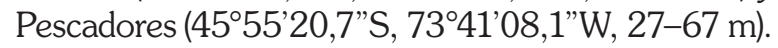

Levinsenia antartica (Strelzov, 1973)

Localidades citadas para Chile:

Región de Magallanes, canal Beaglle, Garibaldi

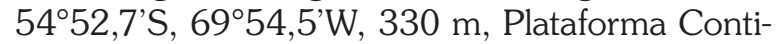
nental de Tierra del Fuego $55^{\circ} 28,8^{\prime} \mathrm{S}, 6^{\circ} 04,4^{\prime} \mathrm{W}$, 1162 m (Montiel et al. 2002).
Presente estudio: fiordo Piti-Palena, sector canal Garrao (4347’59,0”S, 7205’36,0”W, 12-16 m) y río Rodríguez (4347’05,0”S, 7251'11,0”W, 6-10 m). Estero Elefantes, sector San Ramón

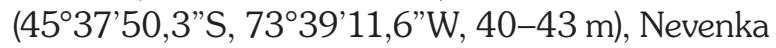
(454'29,6”S, 73³9'07,5'W, 55-126 m) y Pes-

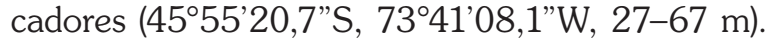
fiordo Aysén, sector caleta Bluff $\left(45^{\circ} 28^{\prime} 41,6^{\prime \prime} \mathrm{S}\right.$, 72 49'54,3"W, 23-26 m), desembocadura río Cuervo (4521'14,4'S, 7303'35,9'W, 45-50 m), punta Tortuga $\left(45^{\circ} 19^{\prime} 28,7^{\prime \prime} \mathrm{S}, 73^{\circ} 05^{\prime} 36,1^{\prime \prime} \mathrm{W}, 33-35 \mathrm{~m}\right)$, caleta Pérez $\left(45^{\circ} 14^{\prime} 34,3^{\prime \prime}\right.$ S, 73¹3'36,9”W, 33-36 m) y Cinco Hermanas (4516’03,7"S, 73¹4'37,9"W, 46-50 m). Fiordo Baker, desembocadura río Baker (4747'53,0”S, 7335'05,6”W, 40-54 m), sector Bajo Pisagua (4747'10,9"S, 7335'20,3”W, 29-48 m) y Punta Raúl (4746’27,1”S, 73³9'15,3”W, 34-66 m).

\section{DISCUSIÓN}

El conocimiento de la riqueza de especies de poliquetos bentónicos en la región de Aisén proviene de trabajos realizados por Wesenberg-Lund (1962) y Hartman-Schröder (1962). Recientemente, estudios sobre la diversidad y composición de poliquetos bentónicos han sido entregados por Cañete et al. (1999) y Rozbaczylo et al. (2005, 2006a, 2006b, 2006c), los cuales han permitido incrementar el número de especies conocidas para la región. De hecho, hasta la fecha se han registrado un total de 91 especies de poliquetos bentónicos entre las zonas intermareales y submareales (Rozbaczylo et al. 2006b). En términos generales, las comunidades de poliquetos de fondos blandos submareales en la región de Aisén se encuentran representadas por 34 familias, siendo las familias Spionidae y Paraonidae las más importantes en términos de riqueza de especies (Tabla 2).

El presente estudio entrega seis nuevos registros de especies de poliquetos bentónicos pertenecientes a la familia Spionidae para la región de Aisén (L. weddellia, S. bombyx, P. ehlersi, R. glutae, $P$. orensanzi y $S$. chilensis) y extiende el rango de distribución para $P$. orensanzi y $S$. chilensis. Por otra parte, se reporta por primera vez la presencia del género Aonides en aguas chilenas. No existen registros previos en aguas chilenas del género y solo se ha registrado Aonides paucibranchiata Southern 1914 en el NE atlántico, Mar del Norte, Mar Negro 
TABLA 2. Lista de especies, localidades, número de especies y familias, y nuevos registros de especies de poliquetos para la región de Aisén

\begin{tabular}{|c|c|c|c|c|c|c|c|c|c|c|c|c|c|c|c|c|c|c|}
\hline Localidad & & Fiord & & & & ordo & Aysé & & & & ero $\mathrm{E}$ & Elefan & & & iordo & Bake & & \\
\hline Sector & 1 & 2 & 3 & 4 & 5 & 6 & 7 & 8 & 9 & 10 & 11 & 12 & 13 & 14 & 15 & 16 & 17 & $\begin{array}{l}\text { Registro en la } \\
\text { región de Aysén }\end{array}$ \\
\hline \multicolumn{19}{|l|}{ Paraonidae } \\
\hline Aricidea antartica & & & & $\mathrm{X}$ & $\mathrm{X}$ & $\mathrm{X}$ & $\mathrm{X}$ & $\mathrm{X}$ & $\mathrm{X}$ & & $\mathrm{X}$ & $\mathrm{X}$ & $\mathrm{X}$ & $\mathrm{X}$ & $\mathrm{X}$ & $\mathrm{X}$ & & No \\
\hline Aricidea strelzovi & & $\mathrm{X}$ & $\mathrm{X}$ & & & & & & $\mathrm{X}$ & $\mathrm{X}$ & $\mathrm{X}$ & $\mathrm{X}$ & $\mathrm{X}$ & & & & & No \\
\hline Aricidea catherinae & & & & & & & & & & $\mathrm{X}$ & & $\mathrm{X}$ & & & & & & No \\
\hline Aricidea albatrossae & & & & & & & & & & & & $\mathrm{X}$ & $\mathrm{X}$ & & & & & No \\
\hline Levinsenia antartica & & $\mathrm{X}$ & $\mathrm{X}$ & & $\mathrm{X}$ & $\mathrm{x}$ & $\mathrm{X}$ & $\mathrm{X}$ & $\mathrm{X}$ & & $\mathrm{X}$ & $\mathrm{X}$ & $\mathrm{X}$ & $\mathrm{X}$ & $\mathrm{X}$ & $\mathrm{X}$ & & No \\
\hline \multicolumn{19}{|l|}{ Spionidae } \\
\hline Laonice weddellia & & & & & $\mathrm{X}$ & & & & & & & & & & $\mathrm{X}$ & & & \\
\hline Spiophanes soederstroemi & $\mathrm{x}$ & $\mathrm{X}$ & & $\mathrm{X}$ & & & $\mathrm{X}$ & & & & & $\mathrm{X}$ & $\mathrm{x}$ & $\mathrm{X}$ & $\mathrm{X}$ & & & $\begin{array}{c}\mathrm{Si} \\
\text { (Rozbaczylo et al. 2006c) }\end{array}$ \\
\hline Spiophanes bombix & & & & & $\mathrm{X}$ & & & & & $\mathrm{X}$ & & $\mathrm{X}$ & & & & & & No \\
\hline Prionospio patagonica & & & & & & & & & & & & & & & & & $\mathrm{X}$ & $\underset{\text { (Augener 1923) }}{\mathrm{Si}}$ \\
\hline Prionospio ehlersi & & & & & & & & & & & & $\mathrm{X}$ & $\mathrm{X}$ & $\mathrm{x}$ & & & & No \\
\hline Prionospio orensanzi & & & & & $\mathrm{X}$ & & $\mathrm{X}$ & & & $\mathrm{X}$ & & $\mathrm{X}$ & & & & & & No \\
\hline Rhynchospio glutaea & & & & & & & & & & & & $\mathrm{X}$ & & & & & & No \\
\hline Scolelepis chilensis & & & & & & & & & & $\mathrm{X}$ & & & & & & & & No \\
\hline Aonides $s p$ & & & & $\mathrm{X}$ & $\mathrm{X}$ & $\mathrm{x}$ & $\mathrm{X}$ & $\mathrm{X}$ & $\mathrm{X}$ & & & & & $\mathrm{X}$ & $\mathrm{X}$ & $\mathrm{X}$ & & No \\
\hline $\begin{array}{l}\text { Número de especies } \\
\text { (Paraonidae-Spionidae) }\end{array}$ & 1 & 3 & 2 & 3 & 6 & 3 & 5 & 3 & 4 & 5 & 3 & 10 & 6 & 5 & 5 & 3 & 1 & \\
\hline $\begin{array}{l}\text { Número de familias de } \\
\text { Polychaeta por sector }\end{array}$ & 5 & 13 & 4 & 17 & 19 & 19 & 18 & 18 & 24 & 17 & 10 & 20 & 11 & 22 & 22 & 19 & 1 & \\
\hline
\end{tabular}

Sectores de estudio: (1) Desembocadura río Palena, (2) Canal Garrao, (3) Río Rodríguez, (4) Bahía Acantilada, (5) Caleta Bluff, (6) Desembocadura río Cuervo, (7) Punta Tortuga, (8) Caleta Pérez, (9) Cinco Hermanas, (10) Paulina, (11) Nevenka, (12) Pescadores, (13) San Ramón, (14) Desembocadura río Baker, (15) Bajo Pisagua, (16) Punta Raúl, (17) Intermareal estuario Baker.

y Argentina (Blake 1983). Los paraonidos constituyen un grupo de poliquetos de pequeño tamaño corporal y usualmente no han sido previamente registrados en la región debido principalmente a la falta de prospecciones bentónicas (Rozbaczylo \& Simonetti, 2000; Montiel et al. 2002). El presente estudio permite extender el rango de distribución hacia el norte de cinco especies (A. antartica, $A$. strelzovi, A. catherinae, A. albatrossea y L. antartica). Los resultados obtenidos en el presente estudio permiten incrementar a 103 especies de poliquetos bentónicos submareales en la región de Aisén, producto de la incorporación de 12 nuevos registros de especies pertenecientes a las familias Spionidae y Paraonidae.

\section{AGRADECIMIENTOS}

El presente estudio fue financiado por los proyectos FONDECYT iniciación en investigación 2007 (Nro. 11075105) y el proyecto FortalecimientoSemilla para Centros Regionales Nro. SS-200805 (CONICYT-CIEP). Fondos adicionales fueron obtenidos del Programa de Financiamiento Basal COPAS Sur- Austral (Nro. PFB 31/2007). P.Ortiz fue financiada por el Programa de Financiamiento Basal COPAS Sur-Austral. Se agradece al proyecto Innova-Chile (Nro. 08CBA-2064) por proveer las muestras del Fiordo Aisén. Nuestros agradecimientos a Juan Ramón Velásquez y Rodrigo Mansilla por su profesional y excelente apoyo durante el trabajo en 
el mar. Especial agradecimiento al Sr. Jorge Arratia y la L/M Halet de Caleta Tortel, Jaime Valencia de la Universidad Austral de Chile, a la tripulación de la L/M Isabeth y al Sr. Jaime Muñoz de la empresa Aquatecma Ltda por proveer las muestras del sector Estero Elefantes.

\section{LITERATURA CITADA}

Bertrán, C., L. Vargas-Chacoff, F. Peña-Cortés, S. Mulsow, J. Tapia, E. Hauenstein, R. Schlatter \& A. Bravo 2006. Macrofauna bentónica de los humedales de tres lagos salinos en el borde costero del sur de Chile. Ciencias Marinas 32(3): 589-596.

Bertrán, C., L. Vargas-Chacoff, F. Peña-Cortés, R. Schlatter, J. Tapia \& E. Hauenstein 2010. Distribución de la macrofauna bentónica en el lago Budi, Sur de Chile. Revista de Biología Marina y Oceanografía 45(2):235-243.

Blake, J. 1983. Polychaetes of the family Spionidae from south America, Antartica and adjacent seas and islands. Antartic Research Series 39(3):205-288.

Buschmann, A.H., V.A. Riquelme, M.C. Hernández- González, D. Varela, J. Jiménez, L.A. Henríquez, P.A. Vergara, R. Guíñez \& L. Filún 2006. A review of the impacts of salmon farming on marine coastal ecosystems in the southeast Pacific. ICES Journal of Marine Science 63, 1338-1345.

Cañete, J.I., G. Leigthon \& F. Aguilera 1999. Polychaetes from Aysén Fjord, Chile: distribution, abundance and biogeographical comparison with the shallow soft-bottom polychaete fauna from Antarctica and the Magellan Province. Scientia Marina 63(Supl.1): 243-252.

Cañete, J.I., B. Hilbig \& M. Santana 2004. Presence of Prionospio (Prionospio) orensanzi Blake, 1983 (Polychaeta: Spionidae) off Punta Arenas, Chile, with notes on their abundance and spatial distribution in shallow, subtidal sandy bottoms. Investigaciones Marinas, Valparaíso 32(2): 121-128.

Carrasco, F. 1974. Spionidae (Polychaeta) provenientes de la Bahía de Concepción y lugares adyacentes. Boletín de la Sociedad de Biología de Concepción 48: 185-201.
Carrasco, F. 1976. Larvas de la Familia Spionidae (Polychaeta) en el plankton de la Bahía de Concepción, Chile. Gayana, Zoología (38): 63pp.

Day, J.H. 1967. A monograph on the Polychaeta of Southern Africa. British Museum Natural History Publication 656: 878 pp.

Ehlers, E. 1901. Die Polychaeten des magellanischen und chilenischen Strandes. Ein faunistischer Versuch. Festschrift zur Feir des Hundertfünfzigjährigen Bestehens der Königlichen Gesellschaft der Wissenschaften zu Göttingen. (abh. Math.-Phys. K), Berlin 232pp.

Fauchald, K. 1977. The polychaeta worms. Definitions and keys to the orders, families and genera. Natural History Museum of Los Angeles County, Science Series 28: 1-190.

Hartman, O. 1964. Polychaeta Errantia of Antartica. Antartic Research Series 3:1-131.

Hartman, O. 1978. Polychaeta from the Weddell Sea Quadrant, Antartica. Antarctic Research Series 26 (4): 125-223.

Hartman, O. 1953. Non-pelagic Polychaeta of Swedish Antarctic Expedition 1901-1903. In Further Zoological Results of the Swedish Antarctic Expedition 1901-1903, 4 (2): 1-83. Hartmann-Schröder, G. 1962. Zur Kenntnis des Eulitorals der chilenischen Pazifikküste und der argentinischen Küste Südpatagoniens unter besonderer Berücksichtigung der Polychaeten und Ostracoden. Tl. II. Die Polychaeten des Eulitorals. Mitteilungen aus dem Hamburgischen Zoologischen Museum und Institut 60 (Suppl.): 57-167.

Hartmann-Schröder, G. 1965. Zur Kenntnis des Sublitorals der chilenischen Küste unter besonderer Berücksichtigung der Polychaeten und Ostracoden. Tl. II. Die Polychaeten des Sublitorals. Mitteilungen aus dem Hamburgischen Zoologischen Museum und Institut 62: 59-305.

Häussermann, V., \& G. Försterra 2009. Fauna marina bentónica de la Patagonia Chilena, Santiago, Nature in Focus, 1000 pp.

Hobson, K. \& K. Banse 1981. Sedentariate and archianelid polychaetes of British Columbia and Washington. Canadian Bulletin of Fisheries and Aquatic Science. Bulletin 209: 1-144. 
Montiel, A., B. Hilbig \& N. Rozbaczylo 2002. New records to Chile of the Family Paraonidae (Annelida: Polychaeta). Helgoland Marine Research (56): 134-139.

Montiel, A., C. Ríos, E. Mutschke \& N. Rozbaczylo 2004. Poliquetos de Fiordos y canales adyacentes al Campo de Hielo Patagínico Sur, Chile (Annelida: Polychatea). Ciencia y Tecnología del Mar 27(1): 49-67.

Montiel, A., D. Gerdes \& W.E. Arntz 2005. Distributional patterns of shallow-water polychaetes in the Magellan region: a zoogeographical and ecological synopsis. Scientia Marina 69 (2): 123-133.

Moreno, R., R. Soto, F. Carrasco \& R. Sepúlveda 2002. Nuevos poliquetos de la familia Spionidae Grube, 1850 (Polychaeta: Spionidae) para el norte de Chile. Noticiario Mensual del Museo Nacional de Historia Natural de Chile 350: 9-11.

Rozbaczylo, N. 1985. Los Anélidos Poliquetos de Chile. Indice Sinonímico y Distribución Geográfica de Especies. Ediciones Pontificia Universidad Católica de Chile. Serie Monografías Biológicas 3:1-284.

Rozbacyzlo, N. \& J. Simonetti 2000. Diversity and distribution of Chilean benthic marine poly- chaetes: State of the art. Bulletin of Marine Science 67(1): 359-372.

Rozbaczylo, N., R. Moreno \& O. Díaz-Díaz 2005. Poliquetos bentónicos submareales de fondos blandos de la región de Aysén, Chile: Clado Phyllodocida (Annelida, Polychaeta). Investigaciones Marinas 33(1): 69-89.

Rozbaczylo, N., R. Moreno, O. Díaz-Díaz \& S.Martínez 2006a. Poliquetos bentónicos submareales de fondos blandos de la región de Aysén, Chile. Clado Terrebellida (Annelida, Polychaeta). Ciencia Tecnología del Mar 29(1):71-90.

Rozbaczylo, N., R. Moreno \& O. Díaz-Díaz 2006b. Poliquetos bentónicos submareales de fondos blandos de la región de Aysén, Chile: Clados Amphimonida, Eunicida, Spionida, Sabellida y Scolecida (Annelida, Polychaeta). Investigaciones Marinas, Valparaíso 34(1): 43-62.

Rozbaczylo, N., R. Moreno \& M. Montes 2006c. Poliquetos bentónicos submareales de fondos blandos desde el seno de Reloncaví hasta la Boca del Guafo, Chile (Annelida, Polychaeta). Ciencia y Tecnología del Mar 29(2): 95-106.

Wesenberg-Lund, E. 1962. Polychaeta Errantia. Reports Lund University Chile Expedition 1948-49. Lunds universitets årsskrift 57(12): 130 pp. 